\title{
AN EQUIPARTITION OF ENERGY PRINCIPLE FOR OSCILLATORY SYSTEMS
}

\author{
P. Rapisarda* J.C. Willems ** \\ * Department of Mathematics, University of Maastricht, P.O. \\ Box 616, 6200 MD Maastricht, The Netherlands, e-mail \\ P.Rapisarda@math.unimaas.nl \\ ** ESAT-SISTA, K.U. Leuven, Kasteelpark Arenberg 10, B-3001 \\ Leuven-Heverlee, Belgium, e-mail \\ Jan.Willems@esat.kuleuven.ac.be
}

\begin{abstract}
We consider oscillatory systems consisting of identical subsystems symmetrically coupled,. We show that the time average of any quadratic functional of the variables of a subsystem and their derivatives equals the time average of the same functional on any other subsystem.
\end{abstract}

Keywords: Behavioral approach; oscillatory systems; quadratic differential form; bilinear differential form; equipartition of energy.

\section{INTRODUCTION}

In this paper we consider oscillatory systems, whose trajectories are linear combinations of sinusoidal functions $w(t)=\sum_{k=1, \ldots, n} A_{k} \sin \left(\omega_{k} t+\right.$ $\left.\phi_{k}\right)$, with $\omega_{k}, A_{k}, \phi_{k} \in \mathbb{R}$ for all $k$. Such are, for example, mechanical systems consisting of connections of a large number of (frictionless) spring and masses, with external variables the displacements or the velocities of the masses from the equilibrium positions; or electrical systems consisting of the interconnection of many inductors and capacitors, with external variables the voltages in the $C$ components or the currents in the $L$ components.

In this paper we state and give a sketch of the proof of a deterministic equipartition of energy principle for oscillatory systems. We prove that if an oscillatory system consists of "symmetrically coupled" (such notion will be formally introduced further in the communication) identical subsystems, then the difference between the value of any quadratic functional of the variables of the one subsystem and their derivatives, and its value on the variables of the other and their derivatives is zero-mean. In particular, the time-averaged total (kinetic+potential) energy of symmetrically coupled undamped oscillators is asymptotically the same for every oscillator. The latter is a result of (Bernstein and Bhat, 2002) which we obtain in a different context and as a special case of a more general result.

We obtain our result using concepts and techniques developed in the behavioral framework (see (Polderman and Willems, 1998)). A special role in our investigation is played by the concept of quadratic differential form $(Q D F)$, introduced in (Willems and Trentelman, 1998).

The paper is structured as follows: after reviewing the basic notions regarding oscillatory systems in the behavioral framework (section 2), we proceed to give an introduction to quadratic differential forms and their calculus (section 3). We then define the notion of conserved- and of zero-mean quantity. Equipped with such notions, we state our equipartition of energy principle. Finally, we show some applications of such principle to simple systems consisting of few oscillators.

The notation used in this paper is standard: the space of $\mathrm{n}$ dimensional real, respectively complex, 
vectors is denoted by $\mathbb{R}^{\mathrm{n}}$, respectively $\mathbb{C}^{\mathrm{n}}$, and the space of $m \times n$ real matrices by $\mathbb{R}^{\mathrm{m} \times \mathrm{n}}$. Whenever one of the two dimensions is not specified, a bullet - is used; so that for example, $\mathbb{R}^{\bullet \times n}$ denotes the set of real matrices with $\mathrm{n}$ columns and an unspecified number of rows. In order to enhance readability, when dealing with a vector space $\mathbb{R}^{\bullet}$ whose elements are commonly denoted with $w$, we use the notation $\mathbb{R}^{\mathrm{w}}$ (note the typewriter font type!); similar considerations hold for matrices representing linear operators on such spaces.

The ring of polynomials with real coefficients in the indeterminate $\xi$ is denoted by $\mathbb{R}[\xi]$; the set of two-variable polynomials with real coefficients in the indeterminates $\zeta$ and $\eta$ is denoted by $\mathbb{R}[\zeta, \eta]$. The space of all $\mathrm{n} \times \mathrm{m}$ polynomial matrices in the indeterminate $\xi$ is denoted by $\mathbb{R}^{\mathrm{n} \times \mathrm{m}}[\xi]$, and that consisting of all $\mathrm{n} \times \mathrm{m}$ polynomial matrices in the indeterminates $\zeta$ and $\eta$ by $\mathbb{R}^{\mathrm{n} \times \mathrm{m}}[\zeta, \eta]$. We denote with $\mathfrak{C}^{\infty}\left(\mathbb{R}, \mathbb{R}^{\mathrm{q}}\right)$ the set of infinitely often differentiable functions from $\mathbb{R}$ to $\mathbb{R}^{q}$.

\section{LINEAR OSCILLATORY BEHAVIORS}

A linear differential behavior is a linear subspace $\mathfrak{B}$ of $\mathfrak{C}^{\infty}\left(\mathbb{R}, \mathbb{R}^{w}\right)$ consisting of all solutions $w$ of a system of linear constant-coefficient differential equations:

$$
R\left(\frac{d}{d t}\right) w=0
$$

where $R \in \mathbb{R}^{\bullet \times w}[\xi]$, is called a kernel representation of the behavior

$$
\mathfrak{B}:=\left\{w \in \mathfrak{C}^{\infty}\left(\mathbb{R}, \mathbb{R}^{\mathrm{w}}\right) \mid w \text { satisfies }(1)\right\},
$$

and $w$ is called the external variable of $\mathfrak{B}$. The class of all such behaviors is denoted with $\mathfrak{L}^{\mathrm{w}}$.

In the following, a special role is played by linear differential autonomous systems. Informally, a system is autonomous if the future of every trajectory in $\mathfrak{B}$ is uniquely determined by its past, equivalently by its present "state" (see (Polderman and Willems, 1998) for a formal definition); in other words, if the system has no inputs. It can be shown that the behavior of an autonomous system admits kernel representations (1) in which the matrix $R$ is square and nonsingular; moreover (see Theorem 3.6.4 in (Polderman and Willems, 1998)), such a representation has the minimal number of equations ( $w$, the number of variables of the system) needed in order to describe an autonomous behavior $\mathfrak{B}$, and is consequently called a minimal representation.

It can be shown that all minimal kernel representations have the same Smith form; for this reason, the diagonal elements in such Smith form are called the invariant polynomials of $\mathfrak{B}$; their product is denoted by $\chi_{\mathfrak{B}}$, and is called the characteristic polynomial of $\mathfrak{B}$. The roots of $\chi_{\mathfrak{B}}$ are called the characteristic frequencies of $\mathfrak{B}$. When considering nonminimal kernel representations, the nonzero invariant polynomials in the Smith form of any matrix $R^{\prime} \in \mathbb{R}^{\bullet \times w}[\xi]$ such that $\mathfrak{B}=\operatorname{ker} R^{\prime}\left(\frac{d}{d t}\right)$, also equal the invariant polynomials of $\mathfrak{B}$ (see Corollary 3.6.3 in (Polderman and Willems, 1998)).

We now define linear oscillatory behaviors.

Definition 1. $\mathfrak{B} \in \mathcal{L}^{\mathrm{w}}$ is an oscillatory behavior if $w \in \mathfrak{B} \Longrightarrow w$ is bounded on $(-\infty,+\infty)$

From the definition it follows immediately that an oscillatory system is necessarily autonomous. The following is a characterization of oscillatory systems in terms of properties of its kernel representation.

Proposition 2. Let $\mathfrak{B}=\operatorname{ker} R\left(\frac{d}{d t}\right)$, with $R \in$ $\mathbb{R}^{\bullet \times w}[\xi]$. Then $\mathfrak{B}$ is oscillatory if and only if every nonzero invariant polynomial of $\mathfrak{B}$ has distinct and purely imaginary roots.

Sketch of proof: We use a classic technique in behavioral system theory, namely reducing the problem to the scalar case by resorting to the Smith form. Without loss of generality assume that the kernel representation induced by $R$ is minimal. Compute the Smith form $R=U \Delta V$ of $R$, with $U, V$ unimodular and $\Delta$ the diagonal matrix of the invariant polynomials $\psi_{i}$ of $R$. With a change of variable, we reduce to the scalar case, namely proving that $\mathfrak{B}_{j}^{\prime}:=\operatorname{ker} \psi_{j}\left(\frac{d}{d t}\right)$ is oscillatory if and only if $\psi_{j} \in \mathbb{R}[\xi]$ has distinct and purely imaginary roots.

(If) Observe that if the characteristic frequencies $\omega_{j k}, k=1, \ldots, \operatorname{deg}\left(\psi_{j}\right)$ of $\mathfrak{B}_{j}^{\prime}$ lie on the imaginary axis and are distinct, then $w_{j}^{\prime} \in \mathfrak{B}_{j}^{\prime}$ if and only if

$$
w_{j}^{\prime}(t)=\sum_{k=1}^{\operatorname{deg}\left(\psi_{j}\right)} \alpha_{j k} e^{i \omega_{j k} t}
$$

for $\alpha_{j k} \in \mathbb{C}, k=1, \ldots, \operatorname{deg}\left(\psi_{j}\right)$. Observe that the $\alpha_{j k}$ 's corresponding to conjugate characteristic frequencies $\pm i \omega_{j k}$ are also conjugate, since each entry of $\psi_{j}(\xi)$ has real coefficients. Conclude that (2) describes a linear combination of sinusoidal functions; thus, $\mathfrak{B}_{j}^{\prime}$ is oscillatory.

(Only if) The proof is by contradiction. Assume that there is a characteristic frequency of $\mathfrak{B}_{j}^{\prime}$ not lying on the imaginary axis; it is easy to verify that this is in contradiction with the boundedness of the trajectories in $\mathfrak{B}_{j}^{\prime}$ on the whole real axis. Now assume by contradiction that there is a characteristic frequency $i \omega_{j k}$ which is not simple. Then there would exist one trajectory $w_{j}^{\prime}$ in $\mathfrak{B}_{j}^{\prime}$ of the form $w_{j}^{\prime}(t)=t \sin \left(\omega_{j k} t+\phi_{j k}\right)$. Since such 
$w_{j}^{\prime}$ is unbounded, this is in contradiction with the oscillatory nature of $\mathfrak{B}_{j}^{\prime}$.

\section{BILINEAR- AND QUADRATIC DIFFERENTIAL FORMS}

A bilinear differential form $(B D F)$ is a functional from $\mathfrak{C}^{\infty}\left(\mathbb{R}, \mathbb{R}^{\mathrm{w}_{1}}\right) \times \mathfrak{C}^{\infty}\left(\mathbb{R}, \mathbb{R}^{\mathrm{w}_{2}}\right)$ to $\mathfrak{C}^{\infty}(\mathbb{R}, \mathbb{R})$, defined as:

$$
L_{\Phi}\left(w_{1}, w_{2}\right)=\sum_{h, k=0}^{N}\left(\frac{d^{h} w_{1}}{d t^{h}}\right)^{T} \Phi_{h, k} \frac{d^{k} w_{2}}{d t^{k}} .
$$

where $\Phi_{h, k} \in \mathbb{R}^{\mathrm{w}_{1} \times \mathrm{w}_{2}}$ and $N$ is a nonnegative integer. Let

$$
\Phi(\zeta, \eta)=\sum_{h, k=0}^{N} \Phi_{h, k} \zeta^{h} \eta^{k},
$$

This two-variable $\mathrm{w}_{1} \times \mathrm{w}_{2}$ polynomial matrix $\Phi(\zeta, \eta)$ induces the bilinear differential form $L_{\Phi}$ defined above.

A BDF $L_{\Phi}$ is symmetric, meaning $L_{\Phi}\left(w_{1}, w_{2}\right)=$ $L_{\Phi}\left(w_{2}, w_{1}\right)$ for all $w_{1}, w_{2}$, if and only if $\Phi$ is a symmetric two-variable polynomial matrix, i.e. if $\mathrm{w}_{1}=\mathrm{w}_{2}$ and $\Phi(\zeta, \eta)=\Phi(\eta, \zeta)^{T}$. The set of symmetric two-variable polynomial matrices of dimension $\mathrm{w} \times \mathrm{w}$ in the indeterminates $\zeta$ and $\eta$ is denoted with $R_{S}^{\mathrm{w} \times \mathrm{w}}[\zeta, \eta]$.

If $\Phi$ is symmetric then it also induces a quadratic functional acting on $\mathfrak{C}^{\infty}\left(\mathbb{R}, \mathbb{R}^{\mathrm{w}}\right)$ as

$$
\begin{aligned}
& Q_{\Phi}: \mathfrak{C}^{\infty}\left(\mathbb{R}, \mathbb{R}^{\mathrm{W}}\right) \rightarrow \mathfrak{C}^{\infty}(\mathbb{R}, \mathbb{R}) \\
& Q_{\Phi}(w):=L_{\Phi}(w, w) .
\end{aligned}
$$

We call $Q_{\Phi}$ the quadratic differential form $(Q D F)$ associated with $\Phi$.

The association of two-variable polynomial matrices with BDF's and QDF's allows to develop a calculus that has applications in many areas of systems and control (see (Willems and Trentelman, 1998) for a thorough exposition). An important role in the following is played by the notion of derivative of a $\mathrm{QDF}$. Given a $\mathrm{QDF} Q_{\Phi}$, we define its derivative as the $\mathrm{QDF} Q_{\dot{\Phi}}$ defined by

$$
Q_{\dot{\Phi}}(w):=\frac{d}{d t}\left(Q_{\Phi}(w)\right)
$$

for all $w \in \mathfrak{C}^{\infty}\left(\mathbb{R}, \mathbb{R}^{w}\right)$. In terms of the twovariable polynomial matrices associated with the QDF's, the relationship between a $\mathrm{QDF} Q_{\Phi}$ and its derivative $Q_{\dot{\Phi}}$ is expressed as

$$
\begin{aligned}
\frac{d}{d t} Q_{\Phi}(w)= & Q_{\dot{\Phi}}(w) \text { for all } w \in \mathfrak{C}^{\infty}\left(\mathbb{R}, \mathbb{R}^{\mathrm{w}}\right) \\
& \Longleftrightarrow \dot{\Phi}(\zeta, \eta)=(\zeta+\eta) \Phi(\zeta, \eta)
\end{aligned}
$$

We end this section with the definition of zeromean quantity.
Definition 3. Let $\mathfrak{B} \in \mathcal{L}^{\text {w }}$ be an oscillatory system, and let $\Phi \in \mathbb{R}_{R}^{\mathrm{w} \times \mathrm{w}}[\zeta, \eta]$. Then $Q_{\Phi}$ is a zeromean quantity for $\mathfrak{B}$ if

$$
w \in \mathfrak{B} \Longrightarrow \lim _{T \rightarrow \infty} \frac{1}{T} \int_{0}^{T} Q_{\Phi}(w)(t) \mathrm{d} t=0
$$

A parametrization of zero-mean QDFs in terms of algebraic properties of the corresponding twovariable polynomial matrices is given next.

Proposition 4. Let $\mathfrak{B} \in \mathcal{L}^{\mathrm{w}}$ be oscillatory, and let $R \in \mathbb{R}^{\mathrm{w} \times \mathrm{w}}[\xi]$ be such that $\mathfrak{B}=\operatorname{ker} R\left(\frac{d}{d t}\right)$. Then $\Phi \in \mathbb{R}_{R}^{\mathrm{W} \times \mathrm{w}}[\zeta, \eta]$ is a zero-mean quantity if and only if there exist $\Psi, X \in \mathbb{R}^{\mathrm{w} \times \mathrm{w}}[\zeta, \eta]$ such that

$$
\begin{aligned}
\Phi(\zeta, \eta)= & (\zeta+\eta) \Psi(\zeta, \eta) \\
& +R(\zeta)^{T} X(\zeta, \eta)+X(\eta, \zeta)^{T} R(\eta)
\end{aligned}
$$

A proof of this result can be found in Proposition 15 of (Rapisarda and Willems, 2004).

In (Rapisarda and Willems, 2004) it is shown that certain zero-mean quantities are such for every oscillatory system, i.e. their zero-mean nature has nothing to do with the dynamics of the particular system at hand, but follows instead from the fact that such quadratic differential forms are derivatives of some other QDF. We call them "trivially zero-mean QDFs"; a parametrization is given in Proposition 22 of (Rapisarda and Willems, 2004).

Example 5. Consider the single oscillator described by the differential equation $m \frac{d^{2} w}{d t^{2}}+k w=0$. The result of Proposition 4 allows us to conclude that the following are zero-mean quantities for $\operatorname{ker}\left(m \frac{d^{2}}{\mathrm{~d} t^{2}}+k\right)$ :

$$
\begin{aligned}
m \zeta \eta-k= & (\zeta+\eta) \underbrace{\frac{1}{2}(\zeta+\eta) m}_{\Psi_{1}} \\
& -\left(m \zeta^{2}+k\right) \cdot \frac{1}{2}-\frac{1}{2} \cdot\left(m \eta^{2}+k\right) \\
(\zeta+\eta) k= & \underbrace{-(\zeta+\eta) m \zeta \eta}_{\Psi_{2}} \\
& +\left(m \zeta^{2}+k\right) \cdot \eta+\left(m \eta^{2}+k\right) \cdot \zeta
\end{aligned}
$$

Observe that the first of these zero-mean quantities is none other than the Lagrangian of the system, while the second one is evidently a trivially zero-mean quantity, being $\frac{d}{d t} k w^{2}$.

\section{A DETERMINISTIC EQUIPARTITION OF ENERGY PRINCIPLE}

We begin this section by formalizing the notion of symmetry in a behavioral framework. As usual, 
we do it in an intrinsic way, i.e. at the level of the trajectories of the behavior (see (Fagnani and Willems, 1993) for a thorough discussion of symmetries and representational issues in a behavioral framework).

Definition 6. Let $\mathfrak{B}$ be a linear differential behavior with w external variables, and let $\Pi \in \mathbb{R}^{\mathrm{w} \times \mathrm{w}}$ be a linear involution, i.e. $\Pi^{2}=I_{\mathrm{w}}$. $\mathfrak{B}$ is called $\Pi$ symmetric if $\Pi \mathfrak{B}=\mathfrak{B}$.

In the following we use the symmetry induced by the permutation matrix

$$
\Pi=\left(\begin{array}{cc}
0 & I_{\mathrm{m}} \\
I_{\mathrm{m}} & 0
\end{array}\right)
$$

or equivalently, we consider systems with $2 \mathrm{~m}$ external variables $w_{i}, i=1, \ldots, 2 \mathrm{~m}$ for which

$$
w \in \mathfrak{B} \Longleftrightarrow\left(\begin{array}{c}
w_{m+1} \\
\vdots \\
w_{2 m} \\
w_{1} \\
\vdots \\
w_{m}
\end{array}\right)=\Pi w \in \mathfrak{B}
$$

We now introduce the notion of observability. Let $\mathfrak{B} \in \mathcal{L}^{\mathrm{w}}$, with its external variable $w$ partitioned as $w=\left(w_{1}, w_{2}\right)$; then $w_{2}$ is observable from $w_{1}$ if for all $\left(w_{1}, w_{2}\right),\left(w_{1}, w_{2}^{\prime}\right) \in \mathfrak{B}$ implies $w_{2}=w_{2}^{\prime}$. Thus, the variable $w_{2}$ is observable from $w_{1}$ if $w_{1}$ and the dynamics of the system uniquely determine $w_{2}$; in other words, the variable $w_{1}$ contains all the information about the trajectory $w=\left(w_{1}, w_{2}\right)$. An algebraic characterization of observability in terms of properties of the matrix $R$ of a kernel representation of $\mathfrak{B}$, and further consequences of this property are given in (Polderman and Willems, 1998).

The main result of this communication is the following.

Theorem $\%$. Let $\mathfrak{B}$ be an oscillatory behavior with $\mathrm{w}=2 \mathrm{~m}$ external variables. Assume that $\mathfrak{B}$ is $\Pi$ symmetric, with $\Pi$ given by (3), i.e. (4) holds. Moreover, assume that

(a) $w_{2}, \ldots, w_{\mathrm{m}}, w_{\mathrm{m}+1}$ observable from $w_{1}$; and

(b) $w_{\mathrm{m}+2}, \ldots, w_{2 \mathrm{~m}}$ observable from $w_{\mathrm{m}+1}$.

Let $\Psi \in \mathbb{R}^{\mathrm{m} \times \mathrm{m}}[\zeta, \eta]$, and consider the $\mathrm{QDF} Q_{\Phi}$ induced by the $2 \mathrm{~m} \times 2 \mathrm{~m}$ two-variable matrix

$$
\Phi(\zeta, \eta):=\left(\begin{array}{cc}
\Psi(\zeta, \eta) & 0 \\
0 & -\Psi(\zeta, \eta)
\end{array}\right)
$$

on $\mathfrak{B}$. Then $Q_{\Phi}$ is a zero-mean quantity for $\mathfrak{B}$.

Sketch of proof: We first reduce ourselves to the case of $\mathrm{w}=2$ in the following way. Symmetry of $\mathfrak{B}$ and observability of $w_{2}, \ldots, w_{m}$ from $w_{1}$, and of $w_{m+2}, \ldots, w_{2 m}$ from $w_{m+1}$ imply that there exists an $F \in \mathbb{R}^{(\mathrm{m}-1) \times 1}[\xi]$ such that

$$
\left(\begin{array}{c}
w_{2} \\
\vdots \\
w_{\mathrm{m}}
\end{array}\right)=F\left(\frac{d}{d t}\right) w_{1} \text { and }\left(\begin{array}{c}
w_{\mathrm{m}+2} \\
\vdots \\
w_{2 \mathrm{~m}}
\end{array}\right)=F\left(\frac{d}{d t}\right) w_{\mathrm{m}+1}
$$

Consequently, $Q_{\Phi}(w)=Q_{\Psi^{\prime}}\left(w_{1}\right)-Q_{\Psi^{\prime}}\left(w_{\mathrm{m}+1}\right)$, where the symmetric two-variable polynomial $\Psi^{\prime}(\zeta, \eta)$ is defined as

$$
\Psi^{\prime}(\zeta, \eta)=\left(\begin{array}{ll}
1 & F^{T}(\zeta)
\end{array}\right) \Psi(\zeta, \eta)\left(\begin{array}{c}
1 \\
F(\eta)
\end{array}\right)
$$

We now prove that the QDF induced by

$$
\left(\begin{array}{cc}
\Psi^{\prime}(\zeta, \eta) & 0 \\
0 & -\Psi^{\prime}(\zeta, \eta)
\end{array}\right) \in \mathbb{R}^{2 \times 2}[\zeta, \eta]
$$

is zero-mean. In order to do so, observe that the projection of $\mathfrak{B}$ on the $w_{1}$ - and $w_{\mathrm{m}+1}$ variable

$$
\begin{aligned}
& \mathfrak{B}_{w_{1}, w_{m+1}}:=\left\{\left(w_{1}, w_{m+1}\right) \mid\right. \exists w_{i}, 2 \leq i \leq 2 m, i \neq 1, m+1 \\
&\text { such that } \left.\left(w_{1}, \ldots, w_{2 m}\right) \in \mathfrak{B}\right\}
\end{aligned}
$$

is oscillatory. Such behavior is symmetric with respect to

$$
J:=\left(\begin{array}{ll}
0 & 1 \\
1 & 0
\end{array}\right)
$$

Using the results of (Fagnani and Willems, 1993) conclude that such a system admits a kernel representation like

$$
\left(\begin{array}{ll}
r_{1} & r_{2} \\
r_{2} & r_{1}
\end{array}\right)
$$

with $r_{i} \in \mathbb{R}[\xi], i=1,2$. Observe that $\operatorname{det}\left(R^{\prime}\right)=$ $r_{1}^{\prime 2}-r_{2}^{\prime 2}$ is an even polynomial, since $\mathfrak{B}$ is oscillatory (see Proposition 2). Conclude from this that $r_{1}^{\prime}$ and $r_{2}^{\prime}$ are even polynomials. Use the fact that the second external variable is observable from the first one to conclude that there exist $a, b \in \mathbb{R}[\xi]$ such that $a r_{1}^{\prime}+b r_{2}^{\prime}=1$. Observe that since $r_{1}^{\prime}$ and $r_{2}^{\prime}$ are even, $a$ and $b$ can also be taken to be even polynomials.

Now let $\Gamma \in \mathbb{R}_{S}[\zeta, \eta]$, and define

$$
X(\xi):=\partial \Gamma(\xi)\left(\begin{array}{cc}
a(\xi) & -b(\xi) \\
b(\xi) & -a(\xi)
\end{array}\right)
$$

It is a matter of straightforward manipulations to see that

$R^{\prime T}(-\xi) X(\xi)+X^{T}(-\xi) R^{\prime}(\xi)=\left(\begin{array}{cc}\partial \Gamma(\xi) & 0 \\ 0 & -\partial \Gamma(\xi)\end{array}\right)$

It can be shown that such equation is equivalent with equation (3); from this we conclude that $Q_{\Gamma}\left(w_{1}\right)-Q_{\Gamma}\left(w_{2}\right)$ is zero-mean. This concludes the proof of the claim.

Example 8. Assume that two equal masses $m$ connected to "walls" by springs of equal stiffness $k$, 
are coupled together with a spring of stiffness $k^{\prime}$. We consider this as the symmetric interconnection, through the spring with elastic constant $k^{\prime}$, of two identical oscillators, each consisting of a mass $m$ and a spring with elastic constant $k$. Take as external variables the displacements $w_{1}$ and $w_{2}$ of the masses from their equilibrium positions; in such case two equations describing the system are

$$
\begin{aligned}
& m \frac{d^{2} w_{1}}{d t^{2}}=k^{\prime}\left(w_{2}-w_{1}\right)-k w_{1} \\
& m \frac{d^{2} w_{2}}{d t^{2}}=k^{\prime}\left(w_{1}-w_{2}\right)-k w_{2}
\end{aligned}
$$

It is easy to verify that these equations describe a symmetric behavior in the sense of Definition 6 .

From the result of Theorem 7 , we can conclude that the difference between the kinetic energies of the two oscillators, represented by the twovariable polynomial matrix

$$
\left(\begin{array}{ll}
m \zeta \eta & 0 \\
0 & -m \zeta \eta
\end{array}\right)
$$

is zero mean. Also the difference between the potential energies of the two oscillators, induced by

$$
\left(\begin{array}{ll}
k & 0 \\
0 & -k
\end{array}\right)
$$

Of course, this implies that on average, also the total energy of the two oscillators is the same.

\section{CONCLUSIONS}

In this communication we have stated the equipartition principle stated in Theorem 7 and given a sketch of its proof. Instrumental in such investigation is the behavioral framework, and the concept of quadratic differential forms. Further results in the direction outlined in this communication can be found in (Rapisarda and Willems, 2004), where also the concept of conserved quantity is discussed, and a decomposition theorem for QDFs is stated.

\section{REFERENCES}

Bernstein, D.S. and S.P. Bhat (2002). Energy equipartition and the emergence of damping in lossless systems. Proc. 41st IEEE CDC pp. 2913-2918.

Fagnani, F. and J.C. Willems (1993). Representations of symmetric linear dynamical systems. SIAM J. Contr. Opt. 31(5), 1267-1293.

Polderman, J.W. and J.C. Willems (1998). Introduction to Mathematical System theory: A Behavioral Approach. Springer-Verlag.

Rapisarda, P. and J.C. Willems (2004). Conserved and zero-mean quadratic quantities in oscillatory systems. Submitted for publication.
Willems, J.C. and H.L. Trentelman (1998). On quadratic differential forms. SIAM J. Control Opt. 36(5), 1703-1749. 